\begin{tabular}{|c|c|c|}
\hline & $\begin{array}{l}\text { European Association for the } \\
\text { Development of Renewable Energies, Environment } \\
\text { and Power Quality (EA4EPQ) }\end{array}$ & $\begin{array}{l}\text { International Conference on Renewable Energies and Power Quality } \\
\text { (ICREPQ'12) } \\
\text { Santiago de Compostela (Spain), 28th to 30th March, } 2012\end{array}$ \\
\hline
\end{tabular}

\title{
Flicker assessment performance of an Electrical Power Standard
}

\author{
J. Bruna, J. M. Castell, D. Cervero, M. A. García, J.J. Melero \\ CIRCE - University of Zaragoza, Zaragoza, Spain \\ C/ Mariano Esquillor Gómez, 15 - Zaragoza 50018 (Spain) \\ Phone/Fax number: +0034 976 762402,e-mail: jbruna@unizar.es / melero@unizar.es
}

\begin{abstract}
This paper presents a flicker compliance testing method useful for the evaluation of electrical power standards. The method uses a very precise self-developed flicker generator based on discrete power semiconductors. $\mathrm{P}_{\text {st }}$, frequency and voltage variations can be accurately compared to assess the performance of flicker generators. A digital flickermeter has also been developed and it is used to obtain the results of the most characteristic flicker parameter, $\mathrm{P}_{\mathrm{st}}$.

With this approach, it is possible to assess the performance of flicker generator standards in consistency with the latest IEC flickermeter testing standard edition.
\end{abstract}

\section{Key words}

Flicker, compliance testing, electrical power standard, voltage variations, digital flickermeter.

\section{Introduction}

Despite the fact that flicker phenomenon does not commonly cause sensitive problems to the devices connected to the grid, this disturbance is getting more important for TSOs because flickering lights can annoy end users. Cyclic connection and disconnection of large loads causing flicker emissions are usually difficult to be minimized. Therefore, flicker emissions and hence their associated voltage variations must be tracked for avoiding health problems or device malfunction.

Flickermeters must be designed following the standards that describe the steps to be performed with the voltage waveform in order to obtain the flicker parameters, Pst and Plt. This is the only way to obtain precise and reliable measurements. A digital flickermeter based on the current standards [1,2] has been developed and it has been used for assessing the performance of an electrical power standard (Fluke 6100A). The results have been compared with those obtained with a semi-ideal flicker generator constructed with discrete power semiconductors.

The flickermeter fulfils all the tests presented in the latest standards, ensuring the validity of the measurements with very low measurement uncertainties. The developed flicker generator, based on a static commutation system, also meets the requirements for high-end power quality standards.
The comparison between the $\mathrm{P}_{\mathrm{st}}$ readings of the two generation methods will demonstrate the validity and accuracy of the Fluke's flicker module for the newer version of the IEC 61000:4-15 standard.

Summarizing, this paper shows preliminary results for the evaluation of flicker measurements by their deviations and uncertainties. The comparison of both methods has been assessed using the criteria of the guideline ILAC G-13:2007 expressing the results using En numbers [3] as well as plots of repeatability standard deviation defined under the ISO 13528:2005 standard [4].

\section{Electrical Power Standard}

The Fluke 6100A is a Power Quality Standard used for calibrating power quality measurement tools to meet the demands of the electrical market. This equipment simulates flicker by amplitude modulating the voltage with depth and modulation frequency set by the user. It can supply any flicker level up to $\pm 30 \%$ of the set voltage within range values $(60 \%$ of $\Delta \mathrm{V} / \mathrm{V})$. Its flexibility allows the user to get flicker waveforms for $120 \mathrm{~V}$ and $230 \mathrm{~V}$ at $50 \mathrm{~Hz}$ and $60 \mathrm{~Hz}$ combinations.

The interface is accessed through a combination of front panel buttons, a mousse and a keyboard. Status information of whole system and current adjusted power quality parameters are showed on the display.

\section{Developed instruments}

The performance of the electrical power standard for flicker generation will be assessed through the comparison of the Fluke 6100A flicker output and the own-developed system with a high-end digital flickermeter. The most relevant characteristics of these three devices will be briefly described next.

\section{$\underline{\text { Digital flickermeter }}$}

The flickermeter architecture was developed following the latest standard for the design of flickermeters [1]. 
This equipment has been accurately characterized for the design nominal values, $120 \mathrm{~V} / 50 \mathrm{~Hz}$. The selected sampling rate for the digital filters and overall statistical evaluation was $36 \mathrm{kS} / \mathrm{s}$. For the evaluation in Block 5 (classifier), 20000 classes were used to obtain the best approach for all the tests.

The equipment meets all the requirements of the current standard [1] with deviations lower than $0.1 \%$ from ideal Pst values. With these results, the flickermeter can be labeled as a Class-A1 instrument for precise and accurate flicker measurements.

\section{Flicker generator}

The system was designed according to the scheme shown in Figure 1.

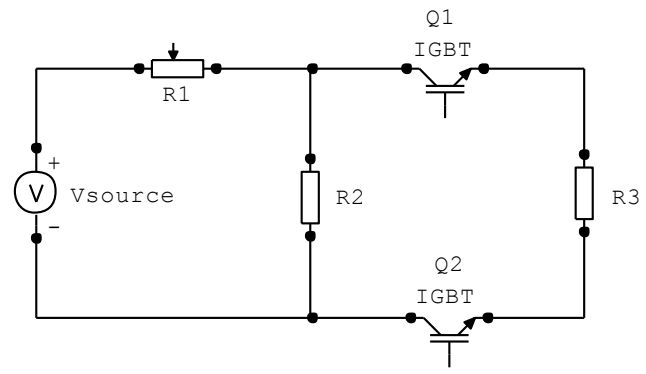

Figure 1. Flicker generator scheme

An Omicron CMC 256 unit is used as a precise voltage source. Its flexibility provides the necessary accuracy $(\varepsilon<$ $0.015 \%$ rd. $+0.005 \%$ rg.) and adjustable voltage output (up to $150 \mathrm{VA}$ ) to obtain the traditional flicker test waveforms [1] with enough stability. For design purposes, this equipment is considered like an ideal voltage source (the current drawn depends only on the circuit to which it is connected).

The flicker modulation depth is obtained by adjusting the 8 W 10-turns potentiometer and the posterior combination of two $50 \mathrm{~W}-1 \mathrm{k} \Omega$ resistors. The two power semiconductor devices (IXSP10N60B2D1) are commanded by a microcontroller to perform a rectangular switching with a $50 \%$ duty cycle. This pulse wave is digitally controlled and calculated by a PIC microcontroller (16F84) through a 4 $\mathrm{MHz}$ crystal with a $\pm 30 \mathrm{ppm}$ frequency tolerance.

Seven switches are used to change the modulation frequency expressed in Changes Per Minute (1CPM, 2CPM, 7CPM, 39CPM, 110CPM, 1620CPM and 4000CPM). A blinking LED mounted on the aluminum enclosure is used to indicate the signal output.

All the tests were performed using $120 \mathrm{~V}$ and $50 \mathrm{~Hz}$ as nominal values. The nominal frequency was chosen the same as the European grid frequency while the $120 \mathrm{~V}$ nominal voltage value was selected due to design restrictions related with the resistor power ratings and the output power limit of the used voltage source.

Figure 2 shows a block diagram of the developed system.

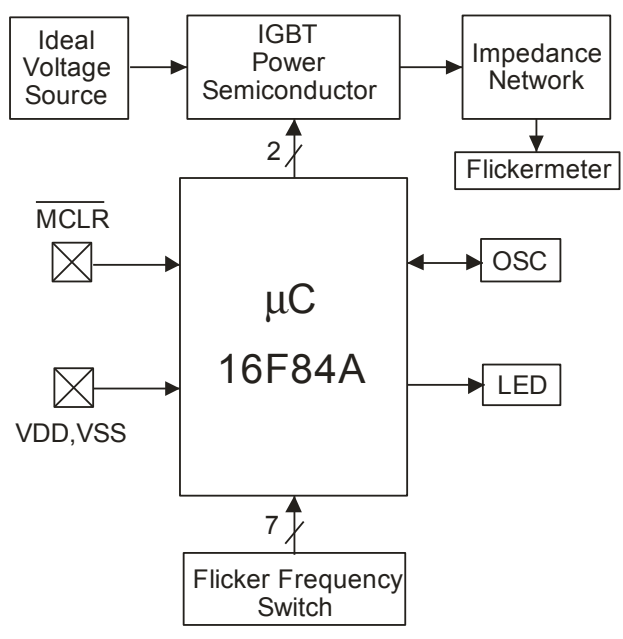

Figure 2. Block diagram of the Flicker generator

\section{Generator performance}

In order to characterize the developed flicker generator, voltage variations $(\Delta \mathrm{V} / \mathrm{V})$ and rectangular modulating frequency were separately assessed. Stability tests were also performed for the equipment.

\section{Modulating Depth}

A calibrated Fluke 8508A DMM (best uncertainty equals to $90 \mu \mathrm{V} / \mathrm{V} \mathrm{rd}$. $+10 \mu \mathrm{V} / \mathrm{V} \mathrm{rg}$.) was used to measure the voltage between high and low states of the rectangular modulation. All voltage measurements for the different tests were made at 1CPM to stabilize the value for being accurately measured by the DMM. No voltage deviations were detected when changing the modulating frequency.

The overall uncertainty was calculated according to the Guide to the Expression of Uncertainty in Measurement [5] taking into account the resolution of the DMM digital indication, the measurement repeatability and the specifications of the measuring instrument.

The obtained results are shown in Table 1.

Table 1. Voltage variation calibration results

\begin{tabular}{|c|c|c|c|}
\cline { 2 - 4 } \multicolumn{1}{c|}{} & Standard [1] & \multicolumn{2}{c|}{ Flicker generator } \\
\hline $\mathbf{C P M}$ & $\boldsymbol{\Delta V / V}(\%)$ & $\Delta \mathbf{V} / \mathbf{V}(\%)$ & $\begin{array}{c}\text { Uncertainty } \\
(\mathbf{\Delta V} / \mathbf{V})(\%)\end{array}$ \\
\hline $\mathbf{1}$ & 3.178 & 3.178 & \pm 0.018 \\
\hline $\mathbf{2}$ & 2.561 & 2.563 & \pm 0.018 \\
\hline $\mathbf{7}$ & 1.694 & 1.694 & \pm 0.018 \\
\hline $\mathbf{3 9}$ & 1.045 & 1.044 & \pm 0.018 \\
\hline $\mathbf{1 1 0}$ & 0.844 & 0.843 & \pm 0.018 \\
\hline $\mathbf{1 6 2 0}$ & 0.545 & 0.548 & \pm 0.018 \\
\hline $\mathbf{4 0 0 0}$ & 3.426 & 3.426 & \pm 0.018 \\
\hline
\end{tabular}


Note that the overall flicker modulation depth accuracy of the Fluke $6100 \mathrm{~A}$ is $\pm 0.025 \%$, comparable to the uncertainty levels shown in Table 1 .

\section{Modulating Frecuency}

The second magnitude that defines a $P_{\text {st }}$ value is the modulating frequency value. To characterize this parameter, a frequency measurement at the IGBT input gate was made. Due to the high switching frequency of the IXSP10N60B2D1 (up to $20 \mathrm{kHz}$ ), the slew rate effect of the transistors in the transitions can be neglected so the output signal exactly matches with the modulation of the flicker voltage waveform. An Agilent 53131A digital counter connected between emitter and gate terminals was used for this purpose. The frecuency calibration results are shown in Table.

Table 2. Frecuency calibration results

\begin{tabular}{|c|c|c|c|}
\cline { 2 - 4 } \multicolumn{1}{c|}{} & Standard [1] & \multicolumn{2}{c|}{ Flicker generator } \\
\hline $\mathbf{C P M}$ & $\begin{array}{c}\text { Frequency } \\
(\boldsymbol{\mu H z})\end{array}$ & $\begin{array}{c}\text { Deviation } \\
(\boldsymbol{\mu H z})\end{array}$ & $\begin{array}{c}\text { Uncertainty } \\
(\boldsymbol{\mu H z} / \mathrm{Hz})\end{array}$ \\
\hline $\mathbf{1}$ & 8333.3 & -0.4 & \pm 8.6 \\
\hline $\mathbf{2}$ & 16666.7 & -0.7 & \pm 8.6 \\
\hline $\mathbf{7}$ & 58333.3 & -5.5 & \pm 8.6 \\
\hline $\mathbf{3 9}$ & 325000.0 & -111.1 & \pm 8.6 \\
\hline $\mathbf{1 1 0}$ & 916666.7 & -798.9 & \pm 8.6 \\
\hline $\mathbf{1 6 2 0}$ & 13500000.0 & -12828.4 & \pm 8.7 \\
\hline $\mathbf{4 0 0 0}$ & 33333333.3 & 6312.9 & \pm 8.6 \\
\hline
\end{tabular}

With the results of previous table, a comparison between the performance of the Power Standard Fluke 6100A and the flicker generator in terms of rectangular modulating frequency accuracy can be represented.

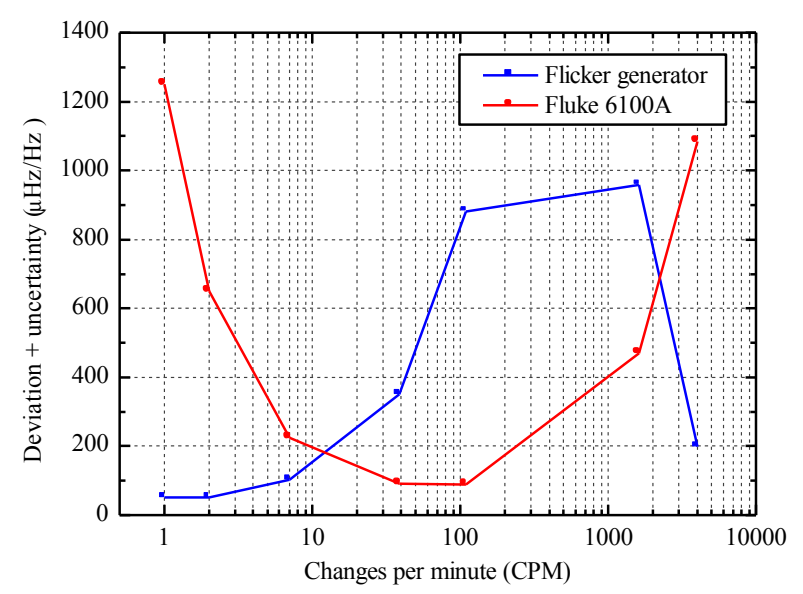

Figure 3. Modulation frecuency comparison

\section{Stability}

Stability performance of the developed flicker generator has been studied and the results are shown in Figure. This test was used to check the system and so its $\mathrm{P}_{\text {st }}$ stability along the time. $400 \mathrm{P}_{\mathrm{st}}$ values were recorded for $1620 \mathrm{CPM}$ and $0.545 \%(\Delta \mathrm{V} / \mathrm{V})$ being relatively constant in the studied interval $\left(\mathrm{P}_{\mathrm{st}}=0.9999 \pm 0.0022\right)$. The inset in Figure 4 shows a detailed view of the $\mathrm{P}_{\mathrm{st}}$ evolution during 2 hours.

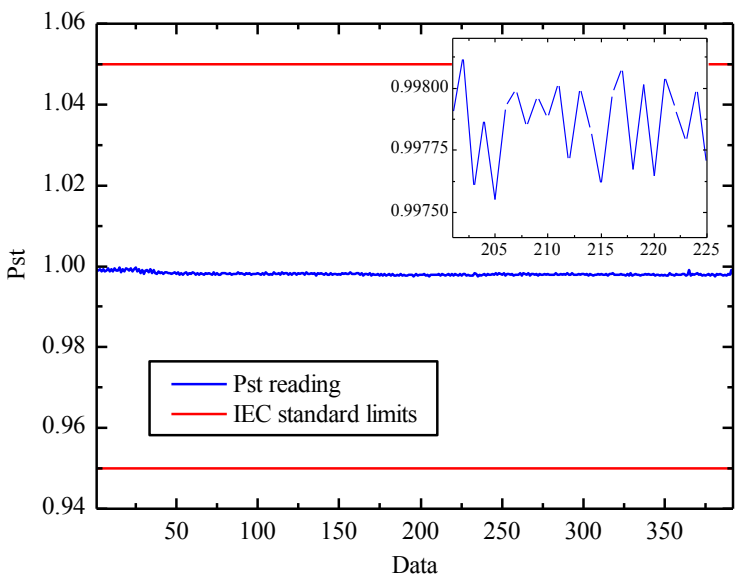

Figure 4. Generation stability test

\section{Results of the comparison}

Seven modulating frequency points were chosen for assessing flicker generation. Modulation voltage depth was selected according to the current standard [1] for obtaining $\mathrm{P}_{\text {st }}$ values equal to 1 . These waveforms were generated with one UKAS certified Fluke 6100A and later with the developed flicker generator. Both outputs were recorded and analysed with the A1-class digital flickermeter. $\mathrm{P}_{\mathrm{st}}$ readings from the two flicker generators were analytically and graphically compared using error bars and En numbers [3].

Several error bars corresponding to the Fluke 6100A and the own-developed flicker generator are represented in the Figure 6. It is clear that the results concerned to Fluke 6100 A completely overlap the $\mathrm{P}_{\text {st }}$ readings from the owndeveloped generator.

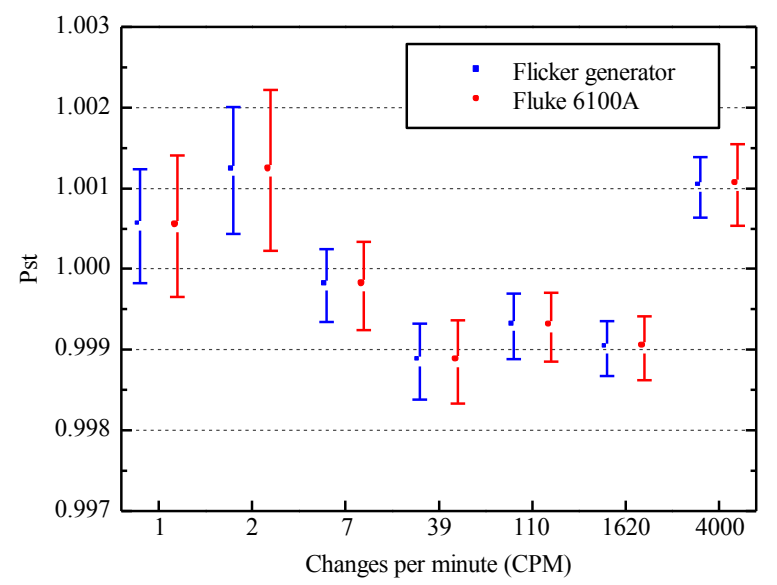

Figure 4. Pst comparative. Fluke 6100A vs flicker generator

The results can also be compared by using the tools applied in interlaboratory comparison, the En numbers [4]. They are calculated with the assigned value determined in a reference laboratory $(\mathrm{X})$, the expanded 
uncertainty of $\mathrm{X}\left(\mathrm{U}_{\mathrm{ref}}\right)$ and the expanded uncertainty of a laboratory's result $\mathrm{x}\left(\mathrm{U}_{\mathrm{lab}}\right)$ according to the following formula:

$$
E_{n}=\frac{x-X}{\sqrt{{U_{L A B}{ }^{2}+U_{R E F}^{2}}^{2}}}
$$

When the expanded uncertainties are calculated using a coverage factor of 2.0, the critical value for an En number is 1.0. En performance levels are normally determined as follows:

$$
\begin{aligned}
& E_{n}<1 \rightarrow \text { Satisfactory performance } \\
& E_{n} \geq 1 \rightarrow \text { Unsatisfactory performance }
\end{aligned}
$$

With these considerations, the En numbers were applied to the comparison. The flicker generator output was considered as the reference value $\mathrm{X}$ and the Fluke 6100A standard output as $\mathrm{x}$ :

$$
E_{n}=\frac{x_{F 6100 A}-X_{\text {Generator }}}{\sqrt{{U_{F 6100 A}^{2}+U_{\text {Generator }}^{2}}^{2}}}
$$

The calculated En numbers are shown in Table 3, being all of them clearly below 1 .

Table 3. Fluke $6100 \mathrm{~A}$ vs developed flicker generator $E_{n}$

\begin{tabular}{|c|c|}
\hline $\mathbf{C P M}$ & $\mathbf{E}_{\mathbf{n}}$ numbers \\
\hline $\mathbf{1}$ & 0.003 \\
\hline $\mathbf{2}$ & 0.001 \\
\hline $\mathbf{7}$ & 0.014 \\
\hline $\mathbf{3 9}$ & 0.005 \\
\hline $\mathbf{1 1 0}$ & 0.031 \\
\hline $\mathbf{1 6 2 0}$ & 0.013 \\
\hline $\mathbf{4 0 0 0}$ & 0.092 \\
\hline
\end{tabular}

\section{Conclusions}

A semi-ideal flicker generator has been developed and tested. The generator was constructed with an ideal voltage source and using a commuted circuit with power semiconductors. It allows obtaining voltage waveforms with user adjustable voltage variation $(\Delta \mathrm{V} / \mathrm{V})$ and frequency modulation.

The flicker generator has been characterized by measuring the voltage variation $(\Delta \mathrm{V} / \mathrm{V})$ and the modulation frequency of its output voltage waveform. The values obtained for both parameters were of the same order of magnitude that those given in the specifications of the Fluke 6100A. The generator stability was also tested by analysing $400 \mathrm{P}_{\text {st }}$ measured values obtained with a modulation frequency of 1620CPM. The obtained $\mathrm{P}_{\mathrm{st}}$ values clearly fulfilled the requirements of the current IEC standard.

A digital Class-A1 flickermeter has been developed. The equipment meets all the requirements of the current standard with deviations lower than $0.1 \%$ from ideal $\mathrm{P}_{\text {st }}$ values.
Finally, the self-made flicker generator and the Power Standard Fluke 6100A have been compared using the $\mathrm{P}_{\text {st }}$ measured at their outputs. The comparison has been performed in two ways, with an error-bar graph and with the En numbers. The obtained results allow concluding that the Fluke 6100A Power Standard complies with the requirements of the IEC 61000-4-15 Standard.

\section{References}

[1] IEC 61000-4-15 ed2.0, "Part 4: Testing and Measurement Techniques - Section 15: Flickermeter Functional and Design Specifications", 2010.

[2] IEC 61000-4-15 ed1.1, "Part 4: Testing and Measurement Techniques - Section 15: Flickermeter Functional and Design Specifications", 2003.

[3] "ILAC G-13: Guidelines for the Requirements for the Competence of Providers of Proficiency Testing Schemes", International Laboratory Accreditation Cooperation, 2007.

[4] "ISO 13528: Statistical Methods for Use in Proficiency Testing by Interlaboratory Comparisons", International Organization for Standarization, 2005.

[5] JCGM 100:2008, "Evaluation of Measurement Data Guide to the Expression of Uncertainty in Measurement", 2008. 